\title{
CARGADOR DE BATERÍAS CON ENERGÍA SOLAR
}

Andrés Fernando Rodríguez*

Javier Andrés Torres****
Jhon Edwin Vera**
Jhon Fredy Bayona***

Sergio Francisco Mora*****

\section{RESUMEN}

El propósito de este trabajo es aprovechar el potencial de energía solar que existe en diferentes zonas de Colombia y los métodos de conversión de energía eléctrica para implementar una aplicación utilizando un panel fotovoltaico y un convertidor reductor topología Buck o reductor síncrono con el fin de cargar baterías entre 3.7V. $6 \mathrm{~V}$ y $12 \mathrm{~V}$ (voltios) para aplicaciones con plataformas móviles y equipos médicos. Se trabaja con un integrado de Texas Instrument que facilita la implementación y la extracción de máxima potencia del panel, analizando los modos de conmutación y los voltajes obtenidos. Debido a la dificultad para la medición de voltaje y corriente en el panel se realizan pruebas de intensidad solar con diferentes tipos de bombillos con el fin de alcanzar los niveles de radiación deseados para voltajes de referencia.

\section{PALABRAS CLAVE}

Energía solar, panel solar, cargador, baterías, equipos móviles, convertidores, fuentes conmutadas, MPPT. 


\begin{abstract}
The purpose of this work is to exploit the potential of solar energy that exists in different areas of Colombia. We want to install an application that charges batteries $3.7 \mathrm{~V}, 6 \mathrm{~V}$ and $12 \mathrm{~V}$ (volts) using the existing methods to convert energy. This application is composed by a photovoltaic panel and a buck converter or reducer synchronous Buck converter.

This can be used with mobile platforms and medical equipment. It works with an integrated Texas Instrument to facilitate the implementation and extraction of maximum power from the panel while analyzing the modes and resultant switching voltages.

Solar intensity tests would be done due to the difficulty of measuring voltage in the panel with different types of lights. This tests would contribute to achieve desired levels of radiation for reference voltages.
\end{abstract}

\section{INTRODUCCIÓN}

La energía emitida por el sol y recibida por la tierra, en un día es diez mil veces superior a la consumida por el planeta durante el mismo. Recientes estudios prevén que durante los próximos años se recibirán cuatro mil veces más energía solar que la que se consumirá. En la actualidad se utilizan baterías recargables en infinidad de dispositivos tales como teléfonos celulares, GPS, equipos para el cuidado de la salud.

Estas baterías proveen los voltajes y corrientes con características en DC necesarias para los circuitos de alimentación en este tipo de aplicaciones; Sin embrago Se hace necesario reabastecer su energía cuando se descargan. Esta situación se agrava en lugares donde el servicio de electricidad es deficiente o nulo.

Debido a que el potencial solar es bueno en gran parte del territorio nacional y que esta energía es limpia e inagotable, se pretende utilizar esta ventaja para la generación de energía eléctrica. Se trabaja con paneles solares y se busca la extracción del máximo punto de potencia o MPPT (seguimiento del punto de máxima potencia), con herramientas y dispositivos que faciliten la aplicación para cargar baterías de $3.7 \mathrm{~V}, 6 \mathrm{~V}$ y $12 \mathrm{~V}$.

\section{METODOLOGÍA Y DESARROLLO}

1.1 Panel fotovolcaico: los paneles fotovoltaicos son los encargados de convertir la energía solar en energía eléctrica. La célula unidad básica de los paneles solares es considerada un dispositivo de dos terminales, que como un diodo conduce la luz que incide sobre este y produce fotovoltaje. Esta foto-voltaje varía entre 0,5 voltios y 1 voltio y una pequeña foto-corriente de décimas de amperio.

Los módulos se arman a partir de la demanda, con varias células en serie (voltaje) y/o en paralelo (corriente) para aumentar sus valores, conformados por cantidades de 26 a 28 células.

De acuerdo con su fabricación se clasificaba en: amorfas y cristalinas. Estas a su vez mono-cristalinas y poli-cristalinas.

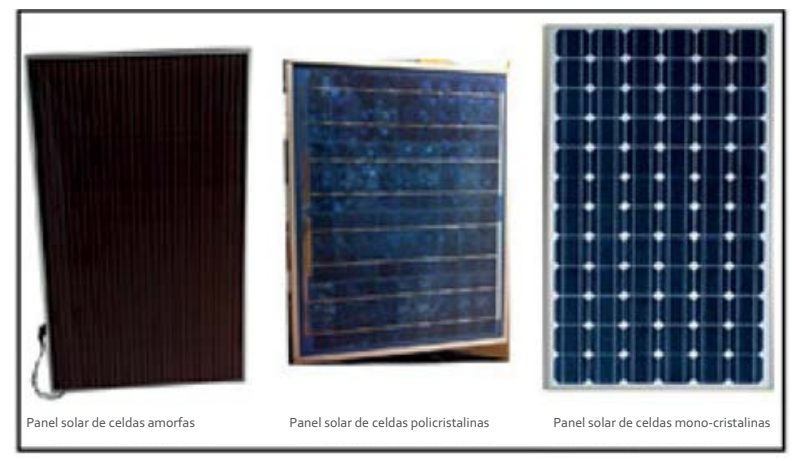

Figura 1: Tipos de paneles Fuente: Elaboración Propia 
1.2 Convertidos reductor, fuentes conmutas: Los convertidores son arreglos de circuitos, generalmente de potencia, que se encargan de la conversión de un nivel de tensión continua a otro, convertidor DCDC. Existen varias topologías utilizadas para elevar o reducir tensión.

Un circuito compuesto por un interruptor, una fuente y una carga (resistencia), puede simular el funcionamiento de una fuente conmutada. En el momento en que se cierra el interruptor, el voltaje Vs (voltaje de la fuente) cae sobre la carga; cuando se abre, el voltaje sobre la carga es cero.

El elemento de conmutación puede ser, un BJT de potencia, MOSFET, IGBT o un GTO. El convertidor reductor o Buck converter, tiene una tensión de salida menor que la tensión de entrada está compuesto por un interruptor electrónico por lo general un MOSFET, un filtro LC pasa bajo y un diodo, (figura 2). Cuando la corriente de la bobina (IL) sea positiva y el diodo este conduciendo se puede definir la corriente permanente, si IL es cero durante el intervalo de conmutación será una IL discontinua.

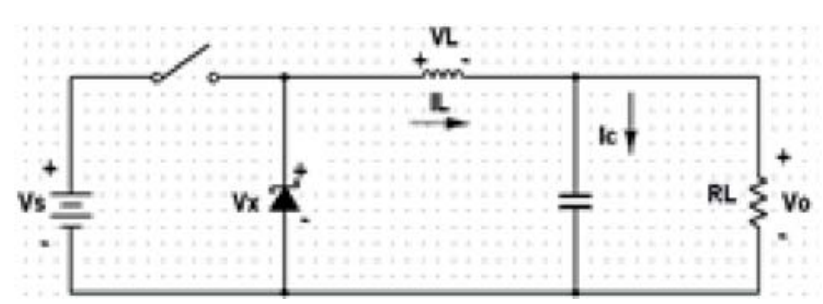

Figura 2: Convertidor reductor Fuente: Elaboración Propia

Condiciones de diseño necesarias con IL permanente:

- La corriente de la bobina debe ser periódica.
- La tensión media en la bobina es cero.

$$
V L=\frac{1}{T} \int_{t}^{t+T} V L(\lambda) d \lambda=0 .
$$

- La corriente media en el condensador es cero.

$$
I C=\frac{1}{T} \int_{t}^{t+T} I C(\lambda) d \lambda=0 .
$$

\section{DESARROLLO DEL PROYECTO}

La figura 3, muestra un reductor síncrono utilizado para el desarrollo de este proyecto; Se diferencia del diseño convencional, en el cual el diodo se cambia por un MOSFET canal N.

Ahora el control del MOSFET principal tiene un pulso complementario el cual se utiliza para activar el segundo MOSFET: Se debe tener cuidado en la activación de los MOSFET, con el fin de evitar las corrientes de corto circuito o "Shoot through currents". Es decir ninguno de los MOSFET se debe encender cuando el otro se encuentre encendido. La ecuación de transferencia de voltaje para el buck convencional es la misma que para un buck síncrono.

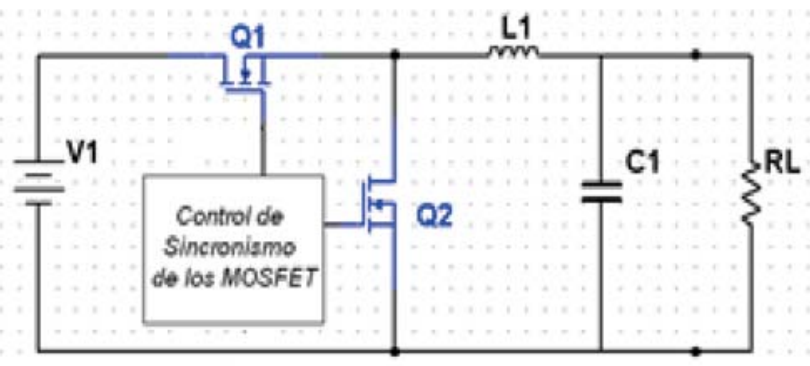

Figura 3. Buck Converter Síncrono. Fuente: Elaboración Propia

$$
I_{L}(t+T)=I_{L}(t) .
$$


Al implementar un cargador de baterías alimentado con energía solar, se deben considerar los valores de trabajo en este caso 12v, 6v y $3,7 \mathrm{v}$.

Los dos primeros valores corresponden a baterías de plomo-acido (Lead acid) y el último valor a batería de Litio-polímero (LiPo). Para cargar la batería LiPo se debe contar con un sistema que corte el suministro de corriente cuando la batería este cargada, para evitar la destrucción de la misma si se le sobrecarga.

La fuente de alimentación debe ser un panel solar de la marca SOLAREX SX 20U, figura 6; con una potencia de salida de 20 watts, voltaje de salida en circuito abierto de $20,1 \mathrm{~V}$, corriente de salida en corto circuito de 1,29 amperios, voltaje de salida a máxima potencia 16,8 v, corriente de salida a máxima potencia 1,19 amperios.

La figura 4 permite observar el circuito a implementar, se debe tener presente que el suministro de energía proviene del panel solar y como controlador es utilizado un circuito integrado $\mathrm{BQ}_{24650}$ de Texas Instrument que cuenta con un módulo específico para el desarrollo de MPPT (Máximum Power Point Tracker) Seguidor del punto de máxima potencia.

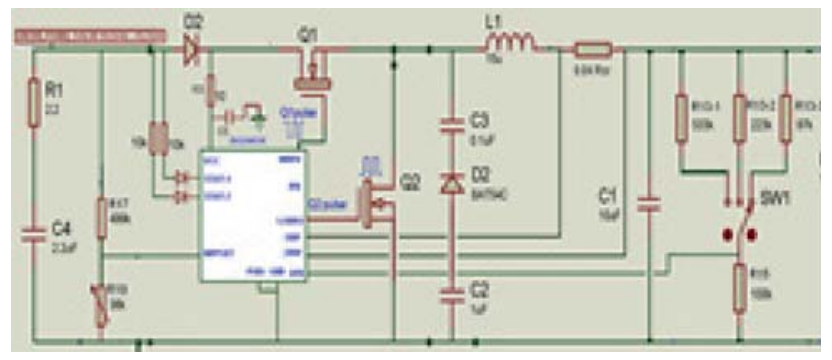

Figura 4. Cargador de baterías, con regulador de carga $\mathrm{BO}_{24650}$ Fuente: Elaboración propia

El circuito implementado se puede definir de la siguiente manera:

1. El filtro $R C$ de entrada, $R 1$ y $C_{4}$, se utiliza para proteger al circuito de los picos de tensión al momento de la conexión a la alimentación, amortiguándolos.
2. El diodo, D2, es para evitar y proteger el terminal VCC de la inversión de voltaje.

3. $\mathrm{R}_{3}$, de $10 \Omega(\mathrm{ohm})$, se encuentra entre el voltaje de entrada y el terminal VCC para filtrar el ruido, junto a este va un condensador, $C_{5}$, con el fin de amortiguar los picos de voltaje.

4. Consta de dos diodos LED con resistencias de 10Kohm, conectados a los terminales STAT1 y STAT2, que indican el estado de la carga de la batería.

5. Para la construcción del Convertidor Reductor Síncrono se utiliza un MOSFET tipo dual canal N, SI7288DP, cuenta con una resistencia en saturación (RDS(ON)) entre 0,022 - 0,019 $\Omega$, un voltaje drainsource (VDS) máximo de $40 \mathrm{~V}$, y una corriente de drain (ID) máxima de 20 Amperios; tienen diodo interno (body diode). El controlador $\mathrm{BO} 24650$ es el encargado de brindar la señal de sincronismo de los GATE en los MOSFET y de regular el ciclo útil, esta señal es de aproximadamente $600 \mathrm{kHz}$.

6. El controlador internamente tiene una señal diente de sierra que es comparada con la señal de control de error EAO, para variar el ciclo útil.

7. Para este circuito el voltaje de la rampa corresponden al $7 \%$ del voltaje de entrada del circuito.

Durante el modo síncrono el controlador asegura que exista una conmutación complementaria a la de los dos MOSFET (break before make) para prevenir que existan corrientes de cortocircuito (Shoot-through).

Los dos MOSFETN son apagados durante un tiempo de $30 \mathrm{~ns}$, lapso requerido por el diodo interno del MOSFETN secundario $\left(\mathrm{Q}_{2}\right)$ para dejar un camino para que la corriente de la bobina siga fluyendo.

Cuando el MOSFET secundario está en conducción hay una menor disipación de potencia que con un diodo Schottky. Durante el modo síncrono existe una corrien- 
te de bobina positiva, corriente permanente, que significa que el Modo de Conducción Continuo (CCM).

8. La señal de sincronismo de los MOSFET se activa en el instante que se detecta la conexión de la batería. Las señales del PWM síncrono, para 12v. Se observa el tiempo de apagado de cada MOSFET al final de cada dutycycle (señal superior MOSFET principal, señal inferior MOSFET secundario).

9. Se utiliza una bobina de $15 \mathrm{uH}$, y un condensador de 1ouF, los cuales se seleccionan respecto a la frecuencia de resonancia del controlador, que está en el rango de $12 \mathrm{kHz}-17 \mathrm{kHz}$, con la siguiente ecuación.

$$
f_{0=\frac{1}{2 \pi \sqrt{L_{0} C_{0}}}}
$$

10. El terminal VFB, es set point de control que utiliza el Cl BQ24650 para realizar la carga de las baterías. Para no tener que utilizar tres circuitos diferentes, se implementan y ajustan tres resistencias variables en $R_{13}$, $\left(R_{13}-1, R 13-2, R 13-3\right)$, en el divisor de VFB, con selectores para cada una; así seleccionar el voltaje de la batería a cargar, esto aplicando la siguiente ecuación.

Con esta se obtuvieron los siguientes valores para R13 se obtuvieron los siguientes valores: $R_{13}-1$ : 533K $\Omega$, R13-2: 223K $\Omega$, R13-3: 97K $\Omega$.

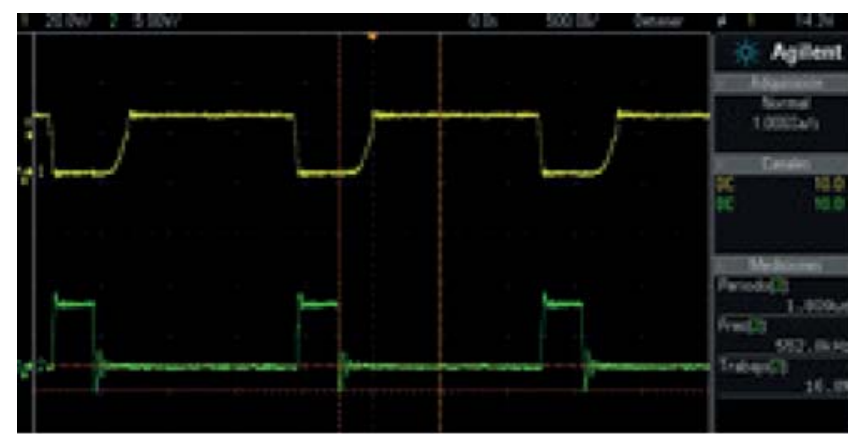

Figura 5. PWM para bacteria de 12 voltios

Fuente: Elaboración propia

\section{RESULTADOS EXPERIMENTALES}

En la tabla 1. Se compara las mediciones realizadas con diferente tipo de bombillos de iluminación artificial halógeno, incandescente y fluorescente.

La altura de exposición y radiación de cada bombillo sobre el panel fue de $25 \mathrm{~cm}$ aproximadamente.

Se realizaron pruebas con cuatro bombillos incandescentes de 60 vatios.

\begin{tabular}{|l|l|c|c|}
\hline Bombillos & $\begin{array}{l}\text { Voltaje del Panel } \\
\text { Voc }\end{array}$ & Bombillos ON & Voltaje del Panel \\
\hline Fluorescente & $7.8 \mathrm{Vdc}$ & 4 & 4 \\
\hline Halógeno & $15.5 \mathrm{Vdc}$ & 3 & 3 \\
\hline Incandescente & $16.4 \mathrm{Vdc}$ & 2 & 2 \\
\hline
\end{tabular}

Fuente elaboración propia

Se aumentaron la cantidad de bombillos y la potencia de estos, la cual varió entre bombillos de 6 y 75 vatios.

$$
\mathrm{V}_{\text {BATT }}=2.1 \mathrm{~V} *\left(1+\left[\frac{\mathrm{R} 13}{\mathrm{R} 15}\right]\right)
$$



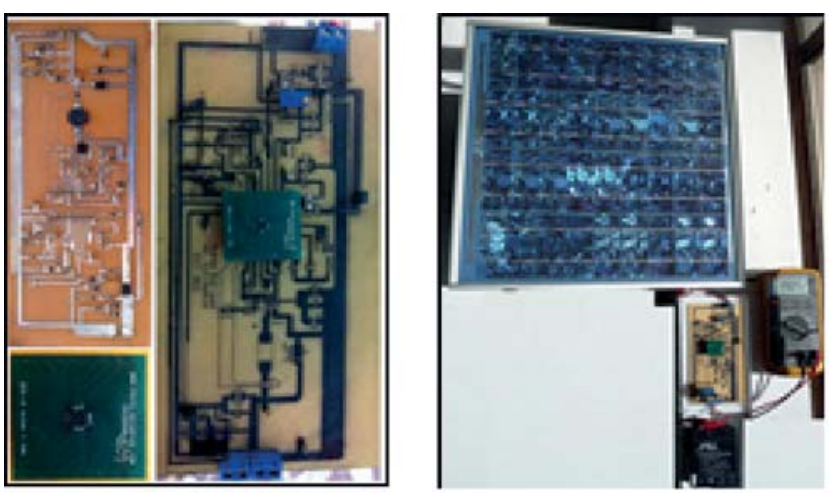

Figura 6. a) Desarrollo de circuito BO24650, b) Panel solar SOLAREX SX Fuente elaboración propio

\section{CONCLUSIONESY FUTUROS TRABAJOS}

El buckconverter síncrono tiene una eficiencia mayor que la del convencional, lo cual supone que la energía suministrada por el panel solar no será disipada en los elementos del circuito.

El controlador conmutado síncrono tiene las características apropiadas para la carga de baterías de LitioPolímero, ya que el suministro de energía termina cuando la batería se encuentra totalmente cargada, lo que prolonga su vida útil.

Las pruebas de iluminación artificial que se realizaron con tres tipos de bombillos mostraron que los bombillos incandescentes eran como mejor alternativa para el análisis sobre el panel.

La estructura lumínica fue hecha con bombillos incandescentes, los cuales generaron demasiado calor, pues aumentaban la temperatura del panel solar y hacían que el MPPT cambiara de posición; era necesario reajustar el divisor de voltaje en el controlador síncrono.

El algoritmo de MPPT del BO presenta un problema comparado con otros algoritmos de MPPT: el de Perturbación Observación (P\&O) y Conductancia Incremental (IncCo), debido a la discontinuidad en el suministro de potencia y a la energía desperdiciada del proceso al enviar la corriente a cero.

Al no existir energía suministrada consecuentemen- te no habrá energía producida, por lo cual siempre se puede alcanzar el punto de máxima potencia.

\section{BIBLIOGRAFÍA}

[1] A W. Leedy, Liping Guo, K A. Aganah, "A constant voltage MPPT method for a solar powered boost converter with DC motor Load" Southeastcon, 2012 Proceedings of IEEE. pp 1-6, 978-1-4673-1375-9/12 2012 IEEE. [ 2 ] H. Rodríguez M .Development of Solar Energy in Colombia and its Prospects. Revista de ingenieria Universidad de los Andes. No 28 pp. 83-87, 2008

[ 3 ] R Faranda, and S Leva, "Energy comparison of MPPT techniques for PV systems", ISSN: 1790-5060, Issue 6, wseas transactions on power systems. Volume 3, pp. 446-455. June 2008.

[ 4 ] E. Trishan, and P. L. Chapman, "Comparison of Photovoltaic Array Maximum Power Point Tracking Techniques," IEEE Transactions on Energy Conversion 22, No. 2 pp.439-449 .June 2007.

[ 5 ] V. Šviković; J. A. Oliver; P. Alou; O. García; J. A. Cobos, "Synchronous buck converter with cutput Impedance correction circuit". Applied power electronics conference and exposition. Vol 1. pp 280-286 Blacksburg, VA 24061 USA 978-1-4577-1216-6/12 @2012 IEEE.

[ 5 ] S Masriand. P Weng Chan, "Development of a microcontroller based boost converter for photo voltaic system," European journal of Scientific research, vol. 41. pp 39-47. 2010.

[6] M Rashid. Electrónica de Potencia. 2a ed. Naucalpan de Juárez, México. Prentice Hall, 1995. ISBN 968880-586-6.

[7] D Hart,. Electrónica de Potencia. Madrid; Prentice 